\title{
Stellarium v0.16.0
}

\section{Georg Zotti \\ Ludwig Boltzmann Institute for Archaeological Prospection and Virtual Archaeology, Vienna, Austria Georg.Zotti@univie.ac.at}

Alexander Wolf

Altai State Pedagogical University, Barnaul, Russia

aw@altspu.ru

The original purpose of the open-source, desktop planetarium Stellarium was to visualise the current skies as realistically as possible. Although it was geared towards contemporary amateur astronomers and laypeople, some of its original features, such as its visual quality and easily exchangeable sky-cultures, as well as its usability and free availability, also made the program very attractive to archaeoastronomers and also cultural astronomers.

As the current main project developers, we gladly accept the JSA editors' invitation to present occasional updates on improvements in the software, especially with respect to historical sky simulation and cultural aspects. In this first Software Note, we want to briefly reflect on the highlights of several recent versions, without going into too much detail. This will also explain why readers should always update Stellarium to the latest version and why authors should always mention the version number when they use Stellarium in their publications: in some places, the original team of developers decided to trade fast computation for some slight inaccuracies which, unfortunately, are mostly apparent in (pre)historical simulation. We have seen some works where authors (and reviewers) may not have been aware of such limitations and we always recommend at least using a second program to verify finds.

With version 0.14 .0 from 24th October, 2015, we introduced precession and ecliptic obliquity from Vondrák et al. (2011), finally fixing the largest astronomical inaccuracy remaining (see Brown 2015). We strongly recommend researchers disregard all previous versions for historical sky simulation (Zotti 2016). Landscape features can now be labelled and the ArchaeoLines plugin (first introduced in version 0.13.1) can show important declination lines (such as solstices, lunar standstills and cross-quarter days) to aid research, especially with the Scenery3D plugin introduced in version 0.13.3. Extinction is now properly dealt with for the Milky Way. Zodiacal light, comet tails and radiants for the 
annual meteor showers are also properly displayed. For telescopic observers, the Deep Sky catalogue has also been greatly extended.

Version 0.15 .0 , issued on 31st July, 2016, introduced a new plugin mostly targeted towards presenters. The RemoteControl plugin makes almost all of Stellarium's interfaces available in a web page controlled in your favourite browser to prevent filling the screen with user interface panels, which allows fully automated set-ups in museums (Zotti et al. 2016). It is now possible to retrieve planet locations from the NASA JPL DE430/DE431 ephemeris files, providing accurate planet locations between $13000 \mathrm{BC}$ and 17000 AD. The ArchaeoLines plugin now displays azimuth lines and geographic targets..

Version 0.15.1, issued on 25th December, 2016, greatly simplifies time zone handling, including Local Mean Solar Time and Local True Solar Time, mostly aimed towards pre-time zone use. A new GUI panel, AstroCalc, provides several computations, such as a conjunction finder. ArchaeoLines can now show arbitrary declinations chosen by the user. Contemporary observers will welcome the DSS layer, which allows for online access to photographic sky coverage.

Version 0.16.0 was released on June 21st, 2017, and introduces a new RemoteSync plugin which allows synchronisation of several systems running Stellarium, e.g. for distributed installations. Minor bodies in the solar system can now be modelled by polygonal irregular shapes. AstroCalc has been greatly extended and can e.g. compute and plot planet ephemerides or phenomena.

Almost every new version also brings new sky-cultures, constellation patterns and artwork from other cultures, contributed by researchers and committed users. We welcome such contributions, especially when they are well researched. We are currently collecting ideas on how to improve the handling of sky-cultures, so please contact us if you are able to contribute. Another community effort is the translation of the software into over 130 languages. Readers can improve Stellarium's translation into their language by joining the effort (see https://translations.launchpad.net/stellarium).

Remaining accuracy issues include accurate orientation of planetary axes, including the Moon. One other component that might undergo revision is the position of fixed stars, since proper motion is presently modelled only with the linear components. 3D correction, including radial velocity, may be aimed at in a future version.

So, if you have not done so, get the latest official version from http://stellarium.org, or even the weekly beta from https://launchpad.net/stellarium, study the PDF manual (Zotti et al., 2017) for details, and stay tuned for more!

\section{References}

Brown, D., 2015. "Exploring Skyscape in Stellarium". Journal of Skyscape Archaeology 1 (1): 93-111. https:// doi.org/10.1558/jsa.v1i1.26956

Stellarium, 2016. Website. Accessed February 2017, http://stellarium.org

Vondrák, J., N. Capitaine and P. Wallace, 2011. "New Precession Expressions, Valid for Long Time Intervals". Astronomy and Astrophysics 534 (A22): 1-19. https://doi.org/10.1051/0004-6361/201117274

Zotti, G., 2016. "Open-source Virtual Archaeoastronomy". Mediterranean Archaeology and Archaeometry 16(4):17-24. https://doi.org/10.5281/zenodo.207260 
Zotti, G., F. Schaukowitsch and M. Wimmer, 2016. "The Skyscape Planetarium". Presentation at the SEAC conference, Bath, 13th September, 2016.

Zotti, G., A. Wolf, M. Gates and B. Gerdes, 2017. Stellarium 0.16.0 User Guide [online] Accessed June 2017, http://sourceforge.net/projects/stellarium/files/Stellarium-user-guide/0.16.0-1/stellarium_user_guide0.16.0-1.pdf/download 